\title{
Quantitative trait analysis and geographic variability of natural populations of Zaprionus indianus, a recent invader in Brazil
}

\author{
JR David ${ }^{1}$, LO Araripe ${ }^{2}$, BC Bitner-Mathé ${ }^{3}, \mathrm{P} \mathrm{Capy}^{1}$, B Goñi ${ }^{4}$, LB Klaczko ${ }^{2}$, H Legout ${ }^{1}$, MB Martins ${ }^{5}$, \\ $\mathrm{J} \mathrm{Vouidibio}^{6}$, A Yassin ${ }^{7}$ and B Moreteau ${ }^{1}$ \\ ${ }^{1}$ CNRS, Lab. Populations, Génétique et Evolution, 91198 Gif sur Yvette Cedex, France; ${ }^{2}$ Departamento de Genetica, IB, Unicamp, \\ Campinas, SP, Brazil; ${ }^{3}$ Departamento de Genetica, Universidade Federal do Rio de Janeiro, Rio de Janeiro 21944-970, Brazil; ${ }^{4}$ Genetica \\ Evolutiva, Facultad de Ciencias, Montevideo 11400, Uruguay; ${ }^{5}$ Museu PE. Goeldi, Belem, Brazil; ${ }^{6}$ Département de Génétique, Université \\ de Brazzaville, Brazzaville, Congo; ${ }^{7}$ Department of Genetics, University of Alexandria, Alexandria, Egypt
}

\begin{abstract}
Five natural samples of a recent South America invader, the drosophilid Zaprionus indianus, were investigated with the isofemale line technique. These samples were compared to five African mainland populations, investigated with the same method. The results were also compared to data obtained on mass cultures of other populations from Africa and India. Three quantitative traits were measured on both sexes, wing and thorax length and sternopleural bristle number. We did not find any latitudinal trend among the American samples, while a significant increase in body size with latitude was observed in the Indian and, to a lesser degree, in the African populations. American populations were also characterized by their bigger size. Genetic variability, estimated by the intraclass correlation among isofemale lines, was similar in American and African populations. The intraline, nongenetic
\end{abstract}

variability was significantly less in the American samples, suggesting a better developmental stability, the origin of which is unclear. A positive relationship was evident between intraline variability of size traits and the wing-thorax length correlation. Altogether, our data suggest that the colonizing propagule introduced to Brazil had a fairly large size, preventing any bottleneck effect being detected. The big body size of American flies suggests that they came from a high-latitude African country. The lack of a latitudinal cline in America seems to be related to the short time elapsed since introduction. The very rapid spread of $Z$. indianus all over South America suggests that it might rapidly invade North America.

Heredity (2006) 96, 53-62. doi:10.1038/sj.hdy.6800753; published online 12 October 2005

Keywords: biological invasion; Drosophila; body size; sternopleural bristles; isofemale lines; wing/thorax ratio

\section{Introduction}

A major and recurrent problem in evolutionary biology is the capacity of natural populations to adapt to local ecological conditions (Hoffmann and Parsons, 1991, 1997; Bijlsma and Loeschcke, 1997; Conner and Hartl, 2004). In this respect, Drosophila species appear as an evolutionary paradigm, since many species have a broad geographic range (David and Tsacas, 1981), live under different climatic conditions and are amenable to a diversity of genetic investigations, including neutral markers, chromosome rearrangements and morphometrical, physiological and behavioural traits.

Temperature is a major factor of the environment, directly responsible for the geographic distribution of many ectotherm species (Andrewartha and Birch, 1954; Precht et al, 1973; Cossins and Bowler, 1987; Leather et al, 1993). Several Drosophila species exhibit latitudinal clines, and genetic variations are strongly correlated with

Correspondence: JR David, Lab. Populations, Génétique et Evolution, CNRS, UPR 9034, Avenue de la Terrasse, Bat 13, BP 1 91198, Gif sur Yvette Cedex, France. E-mail: david@pge.cnrs-gif.fr

Received 30 March 2005; accepted 10 August 2005; published online 12 October 2005 local climatic conditions especially temperature. A major attention has been paid to body size, with the recurrent observation that size increases in colder climates (Capy et al, 1993; Partridge and French, 1996; Van Voorhies, 1996; Atkinson and Sibly, 1997; Partridge and Coyne, 1997; Blackburn et al, 1999; Angilletta and Dunham, 2003). Size is not however the only trait that exhibits latitudinal clines, and many others are documented, at least in the two most investigated species, Drosophila melanogaster and D. simulans (David and Capy, 1988; Capy et al, 1993; David et al, 2004; Gibert et al, 2004).

Even when selective mechanisms are suspected, the exact targets of selection are not precisely identified, one reason being that comparisons concern long-established populations with an unknown history of geographic extension. Recently introduced species offer a rare and unique opportunity to follow the adaptive process 'in flagrante delicto'. In this respect, the introduction of the cold-adapted, European D. subobscura in South and North America more than 20 years ago has been a most interesting and well-investigated example of progressive adaptation (Brncic et al, 1981; Prevosti et al, 1985; Ayala et al, 1989; Huey et al, 2000; Santos et al, 2005).

We consider now a second analogous and more recent case: the colonization of the South-American continent 
by an Afrotropical drosophilid, Zaprionus indianus. The first record of Z. indianus in Brazil was in late 1998 (Vilela, 1999) and, since this date, the species has increased its range very rapidly, being now found from the Equator (Belem) up to Uruguay (Goñi et al, 2001). Z. indianus is now very common in cultures and domestic habitats, and may be a pest for fig cultures (Vilela, 1999; Tidon et al, 2003).

Z. indianus is a typical Afrotropical species (Chassagnard and Kraaijeveld, 1991) and more than 10 related species are endemic to that region. It was however first described from India (Gupta, 1970), where it has been introduced at an unknown date. The geographic variability of Indian populations has been analysed and several latitudinal clines have been described (Karan et al, 1998a, 2000). The African, ancestral populations have not, however, been investigated so far.

In the present paper, we used the isofemale line technique (Hoffmann and Parsons, 1988; David et al, 2005) for a precise analysis of South-American populations collected between 1999 and 2004, and spanning a latitudinal range of $35^{\circ}$. A comparison is made with recently collected African populations, analysed with the same procedure. The comparison is extended to results obtained on older laboratory strains from Africa and India. Two kinds of unrelated traits have been investigated: body size and thoracic bristle number. The main result is that American populations are characterized by their large body size, without any latitudinal trend, while latitudinal clines may be observed in Africa and India.

\section{Materials and methods}

\section{Populations investigated}

We investigated five South-American natural populations collected between December 1999 and December 2004 (see Table 1). These populations were analysed with the isofemale line technique, in their second laboratory generation (David et al, 2005). The same procedure was applied to four African localities, on populations sampled between 2000 and 2003 (Table 1) for a total of 70 lines.

We also investigated a set of seven Old-World populations, kept as mass cultures, generally for several years, under laboratory conditions (see Table 7). Finally, we also compared the data to those previously published (Karan et al, 2000) on 10 latitudinal populations from India.

\section{Experimental procedure}

For the populations analysed with the isofemale line technique, we isolated single wild collected females in culture vials for the production of F1 progeny. A set of 10 pairs from each line was then randomly taken as parents for the experimental flies. After about a week at $20-22^{\circ} \mathrm{C}$ (a delay necessary for Zaprionus adults to reach their reproductive maturity), each parental group was transferred to a culture vial containing a killed yeast, high nutrient medium (David and Clavel, 1965) and the operation was repeated for 2 successive days.

For D. melanogaster, this medium prevents any significant variation of morphometrical traits according to larval density (Karan et al, 1999b). Such is not the case for $Z$. indianus. This species is far more sensitive to larval density and, when many larvae are present in a culture, they try to escape and pupate outside the vials. This results in a high pupal mortality and, more importantly, in a heterogeneity between developmental conditions among individuals. As a remedy, we strongly controlled larval density in each vial. When a vial appeared to harbour too many young larvae, part of them were transferred to a fresh culture vial. With that procedure, larval density was kept below 50 larvae per vial, and most pupae remained at the food level. After removing the parents, experimental vials were transferred at $25^{\circ} \mathrm{C}$.

Table 1 Mean values of morphometrical traits in natural populations of South America or Africa investigated with the isofemale line technique

\begin{tabular}{|c|c|c|c|c|c|c|c|c|c|c|}
\hline \multirow[t]{2}{*}{ Population } & \multirow[t]{2}{*}{$\mathrm{n}$} & \multirow[t]{2}{*}{ Latitude } & \multicolumn{2}{|c|}{ Wing length } & \multicolumn{2}{|c|}{ Thorax length } & \multicolumn{2}{|c|}{ Wing/thorax ratio } & \multicolumn{2}{|c|}{ Sternopleural bristles } \\
\hline & & & Female & Male & Female & Male & Female & Male & Female & Male \\
\hline \multicolumn{11}{|l|}{ South America } \\
\hline Rio, 1999 & 23 & $22.6 \mathrm{~S}$ & $300.97 \pm 0.47$ & $296.23 \pm 0.54$ & $137.98 \pm 0.24$ & $137.33 \pm 0.24$ & $2.181 \pm 0.003$ & $2.157 \pm 0.003$ & $19.58 \pm 0.24$ & $18.61 \pm 0.23$ \\
\hline Salvador, 1999 & 10 & $13.0 \mathrm{~S}$ & $301.08 \pm 1.47$ & $293.92 \pm 1.04$ & $137.34 \pm 0.61$ & $135.96 \pm 0.41$ & $2.192 \pm 0.01$ & $2.162 \pm 0.01$ & $19.73 \pm 0.26$ & $18.43 \pm 0.17$ \\
\hline Rio, 2000 & 11 & $22.6 \mathrm{~S}$ & $297.35 \pm 0.93$ & $291.93 \pm 0.96$ & $137.47 \pm 0.56$ & $136.35 \pm 0.67$ & $2.163 \pm 0.01$ & $2.142 \pm 0.01$ & $19.45 \pm 0.40$ & $18.36 \pm 0.29$ \\
\hline Belem, 2002 & 11 & $1.3 \mathrm{~S}$ & $295.09 \pm 1.40$ & $289.93 \pm 1.21$ & $137.15 \pm 0.53$ & $136.36 \pm 0.46$ & $2.152 \pm 0.01$ & $2.126 \pm 0.01$ & $19.25 \pm 0.38$ & $18.32 \pm 0.29$ \\
\hline Montevideo, 2004 & 20 & $34.5 \mathrm{~S}$ & $296.78 \pm 0.86$ & $291.28 \pm 0.90$ & $136.75 \pm 0.35$ & $135.55 \pm 0.41$ & $2.170 \pm 0.004$ & $2.149 \pm 0.004$ & $19.72 \pm 0.19$ & $18.39 \pm 0.23$ \\
\hline Mean & & & $298.25 \pm 1.19$ & $292.66 \pm 1.10$ & $137.34 \pm 0.20$ & $136.31 \pm 0.30$ & $2.172 \pm 0.007$ & $2.147 \pm 0.006$ & $19.55 \pm 0.09$ & $18.42 \pm 0.05$ \\
\hline \multicolumn{11}{|l|}{ Africa } \\
\hline Sao Tome, 2001 & 11 & $0.2 \mathrm{~N}$ & $277.53 \pm 1.10$ & $272.04 \pm 1.55$ & $132.04 \pm 0.45$ & $130.65 \pm 0.57$ & $2.102 \pm 0.005$ & $2.082 \pm 0.006$ & $19.55 \pm 0.54$ & $18.09 \pm 0.37$ \\
\hline Sao Tome, 2003 & 15 & $0.2 \mathrm{~N}$ & $272.88 \pm 0.89$ & $268.35 \pm 0.78$ & $130.67 \pm 0.49$ & $129.13 \pm 0.35$ & $2.089 \pm 0.010$ & $2.078 \pm 0.01$ & $20.09 \pm 0.33$ & $18.57 \pm 0.27$ \\
\hline Benin, 2000 & 10 & $6.2 \mathrm{~N}$ & $282.24 \pm 0.78$ & $277.24 \pm 0.71$ & $129.88 \pm 0.64$ & $128.36 \pm 0.55$ & $2.174 \pm 0.008$ & $2.161 \pm 0.008$ & $20.54 \pm 0.26$ & $18.87 \pm 0.27$ \\
\hline Cape Town, 2002 & 12 & $33.6 \mathrm{~S}$ & $289.10 \pm 0.92$ & $283.87 \pm 1.07$ & $132.00 \pm 0.38$ & $130.97 \pm 0.41$ & $2.190 \pm 0.005$ & $2.168 \pm 0.005$ & $19.51 \pm 0.38$ & $18.39 \pm 0.33$ \\
\hline Madagascar, 2003 & 22 & $18.5 S$ & $287.49 \pm 0.93$ & $283.15 \pm 0.93$ & $132.35 \pm 0.42$ & $131.33 \pm 0.35$ & $2.173 \pm 0.006$ & $2.157 \pm 0.006$ & $20.58 \pm 0.25$ & $19.64 \pm 0.21$ \\
\hline Mean & & & $281.85 \pm 3.03$ & $276.93 \pm 3.04$ & $131.39 \pm 0.48$ & $130.09 \pm 0.57$ & $2.146 \pm 0.021$ & $2.129 \pm 0.020$ & $20.05 \pm 0.23$ & $18.71 \pm 0.26$ \\
\hline Total mean & & & $290.05 \pm 3.14$ & $284.79 \pm 3.03$ & $134.36 \pm 1.02$ & $133.2 \pm 1.08$ & $2.159 \pm 0.011$ & $2.138 \pm 0.010$ & $19.80 \pm 0.14$ & $18.57 \pm 0.14$ \\
\hline $\begin{array}{l}\text { Intercontinental } \\
\text { comparison }(t \text {-test) }\end{array}$ & & & $5.04^{* *}$ & $4.87^{* *}$ & $11.53^{* * *}$ & $9.67^{* * *}$ & $1.19 \mathrm{~ns}$ & $0.85 \mathrm{~ns}$ & $2.05 \mathrm{~ns}$ & $1.08 \mathrm{~ns}$ \\
\hline
\end{tabular}

$n=$ number of lines. Lengths are in $\mathrm{mm} \times 100$. Comparisons of the two geographic groups are made with Student's $t$-test $(n=5$ in each group). Level of significance: ns: non significant; ${ }^{* *} P<0.01$; ${ }^{* * *} P<0.001$. 
After 13 days, emerged adults were stored on fresh food, waiting to be measured. The number of available isofemale lines was quite variable across populations, and ranged between 10 and 23 . These numbers are given in Table 1, for an overall total number of 145 lines in 10 different populations.

\section{Morphometrical analysis}

Adults from the same line but developed in different vials were pooled into a single sample, and 10 flies of each sex were randomly taken for being measured after anaesthesia. Two size-related traits were investigated: the total wing length, from the thoracic articulation to the tip; the total thorax length, from the neck to the tip of the scutellum. Measurements were performed with a micrometer in a binocular microscope, and micrometer units converted to $\mathrm{mm} \times 100$. As in previous papers, we also calculated the wing/thorax ratio (W/T ratio), which appears as a specific trait, related to wing loading and flight capacity (Pétavy et al, 1997). A meristic trait, sternopleural bristle (STP) number, was also measured on the right and left side of the thorax, and then the sum was calculated.

\section{Data analysis}

Data were analysed with classical statistical methods, using the Statistica package (1999). We considered not only the mean values of the traits, but also their variability, expressed as a coefficient of variation $(\mathrm{CV})$ both at the intra- and between-line level. Genetic variability was appreciated by calculating a coefficient of intraclass correlation (ICC) and a genetic CV (evolvability; Hoffmann and Parsons, 1988; Houle, 1992; David et al, 2005). Phenotypic (intraline) and genetic (betweenline) correlations were also investigated between different traits. Finally, we also analysed the relationships between morphometrical traits and latitude of origin in the various geographic groups.

\section{Results}

\section{Mean values in populations analysed by isofemale technique}

Mean values of morphometrical traits are given in Table 1 for the five South-American samples, collected between 1999 and 2004, and for five Afrotropical populations, collected during the same period. Results of ANOVA applied for each trait to the whole data set of 10 samples are shown in Table 2.

For the two size-related traits, there is a significant difference between continents, more pronounced for thorax length. A similar conclusion is provided $t$-tests in Table 1: American populations are bigger than African ones. For the $\mathrm{W} / \mathrm{T}$ ratio, the intercontinental effect disappears. For STP bristles, there is a slight difference between continents, but in females only. For all traits, except STP bristles in females, there is a highly significant population effect, which means a heterogeneity among populations of the same continent. This phenomenon was further investigated by separate ANOVAs (not shown) within each continent. The heterogeneity between populations was always greater among African populations than among American ones. The phenomenon was especially visible for $\mathrm{W} / \mathrm{T}$ ratio: in
Table 2 Results of ANOVA on whole data set

\begin{tabular}{lcccc}
\hline Trait & Continent & Population & Line & Residual \\
\hline d.f & 1 & 8 & 135 & 13.05 \\
Wing female & $35.19^{*}$ & $35.40^{* * *}$ & $9.86^{* * *}$ & 19.55 \\
Wing male & $34.68^{*}$ & $35.78^{* * *}$ & $10.65^{* * *}$ & 18.88 \\
Thorax female & $32.32^{* *}$ & $19.23^{* * *}$ & $15.10^{* * *}$ & 33.35 \\
Thorax male & $34.91^{* *}$ & $20.01^{* * *}$ & $12.88^{* * *}$ & 32.20 \\
W/T ratio female & $4.77 \mathrm{~ns}$ & $39.83^{* * *}$ & $19.51^{* * *}$ & 35.90 \\
W/T ratio male & $1.97 \mathrm{~ns}$ & $35.85^{* * *}$ & $18.81^{* * *}$ & 43.36 \\
STP female & $1.74^{*}$ & $1.96 \mathrm{~ns}$ & $54.39^{* * *}$ & 41.91 \\
STP male & $0.43 \mathrm{~ns}$ & $4.63^{* * *}$ & $23.09^{* * *}$ & 71.86 \\
\hline
\end{tabular}

The table shows the percentage of the total variance explained by continents, populations (nested in continents), lines (nested in populations) and individual flies (residual). Level of significance: ns: nonsignificant; ${ }^{*} P<005$; ${ }^{* *} P<0.01$; ${ }^{* * *} P<0.001$. d.f: degrees of freedom.

America, variations among populations accounted for $10.2 \%$ of the total variation, while the proportion amounted to $51.1 \%$ in Africa. Looking at Table 1, we see that the samples from Sao Tome are remarkable by a low ratio (2.1) corresponding to a high wing loading.

In all cases, we found a highly significant line effect, indicating, as expected, an intrapopulation genetic variability. The amount of the total variation explained at this level is however variable among traits, from $9.9 \%$ for female wing up to $54.4 \%$ for female STP number.

The bigger size of Brazilian flies is illustrated in Figure 1, which compares the Rio samples with the smallest African population from Sao Tome island. We see that the best discrimination is provided by wing length, since it is possible to assign, without any ambiguity, each isofemale line to its geographic origin. The discriminating power is slightly less for thorax length, since there is a slight overlap between the two distributions. Both size traits exhibit a clear positive correlation between female and male values, as illustrated in Figure 1.

\section{Variability within isofemale lines}

Intraline CVs: For each trait, the variability among the 10 flies measured in each isofemale line was characterized by its $\mathrm{CV}$, for a total number of 145 lines. Mean values observed in the different populations are given in Table 3. For all traits (except W/T ratio). ANOVA (not shown) revealed a significant heterogeneity among the 10 populations. This phenomenon was further explored by comparing the two geographic groups either with ANOVA or with a Student's $t$-test based on the mean values of the 10 populations. Both methods led to the same conclusion: significant differences were found between the two groups, except for the $\mathrm{W} / \mathrm{T}$ ratio in both sexes and the bristle number in males. In all significant cases, American populations were consistently less variable than African ones.

Comparisons among sexes failed to evidence any significant difference between males and females. There were however significant differences between traits: wing length was slightly less variable than thorax length, on average 1.71 vs 1.84 ; the $\mathrm{W} / \mathrm{T}$ ratio was less variable than the traits themselves (on average 1.42). Finally, the sternopleural bristle number was much more variable (on average 9.18), as usual for meristic traits. 

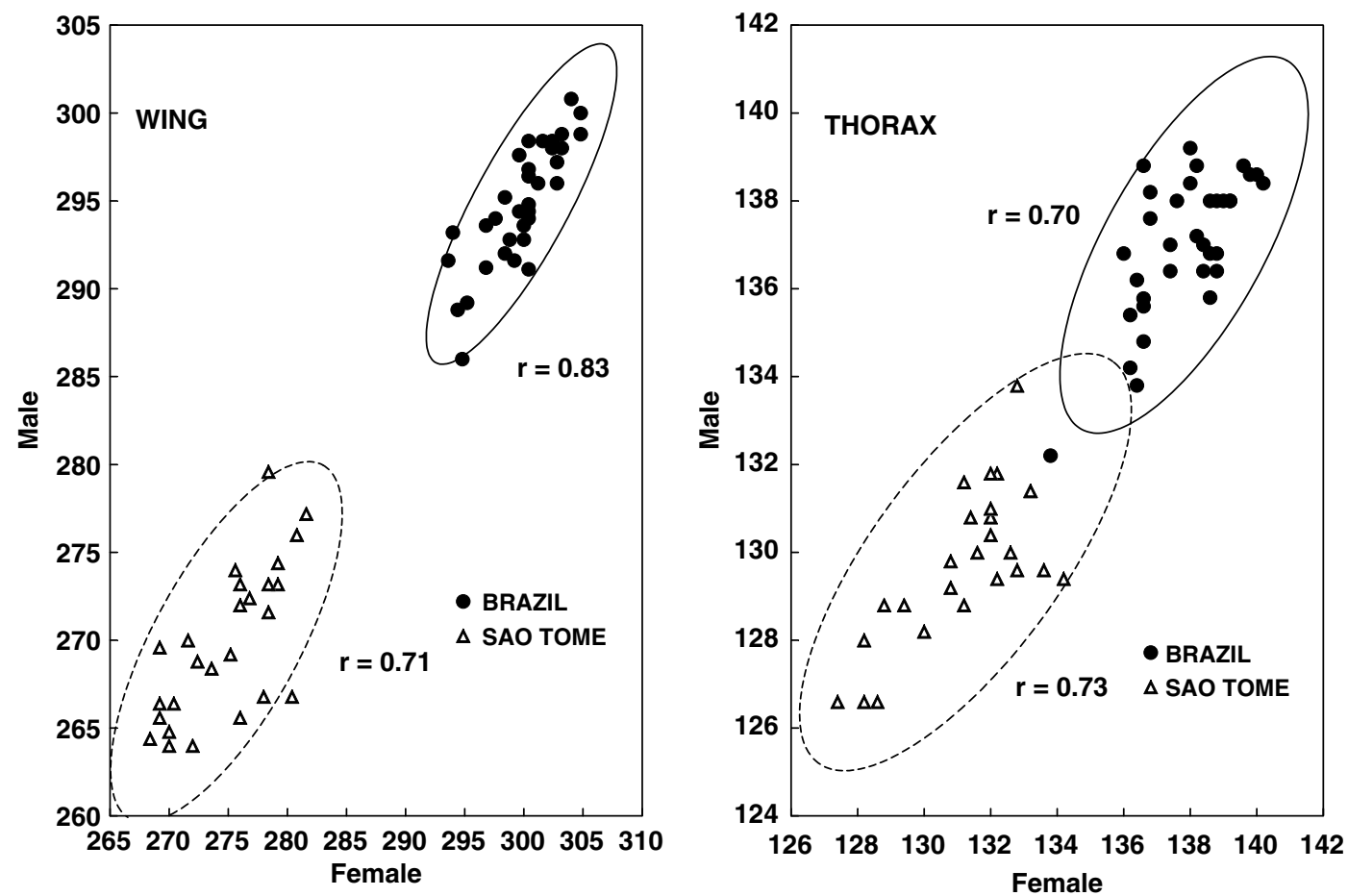

Figure 1 Difference in body size traits between Brazilian population from Rio ( $n=34$ isofemale lines) and Sao Tome population ( $n=26$ lines). Each symbol is the mean value of an isofemale line. $r=$ correlation between females and males.

Table 3 Analysis of the intraline variability

\begin{tabular}{|c|c|c|c|c|c|c|c|c|}
\hline \multirow[t]{2}{*}{ Population } & \multicolumn{2}{|c|}{ Wing length } & \multicolumn{2}{|c|}{ Thorax length } & \multicolumn{2}{|c|}{ Wing/thorax ratio } & \multicolumn{2}{|c|}{ Sternopleural bristles } \\
\hline & Female & Male & Female & Male & Female & Male & Female & Male \\
\hline Rio, 1999 & $1.37 \pm 0.08$ & $1.46 \pm 0.10$ & $1.7 \pm 0.10$ & $1.63 \pm 0.10$ & $1.44 \pm 0.06$ & $1.35 \pm 0.07$ & $7.47 \pm 0.40$ & $8.81 \pm 0.47$ \\
\hline Salvador, 1999 & $1.66 \pm 0.10$ & $1.46 \pm 0.13$ & $1.42 \pm 0.09$ & $1.50 \pm 0.13$ & $1.35 \pm 0.09$ & $1.19 \pm 0.11$ & $7.37 \pm 0.85$ & $7.86 \pm 0.68$ \\
\hline Rio, 2000 & $1.60 \pm 0.16$ & $1.47 \pm 0.09$ & $1.69 \pm 0.13$ & $1.72 \pm 0.13$ & $1.50 \pm 0.08$ & $1.45 \pm 0.10$ & $8.73 \pm 0.53$ & $9.64 \pm 0.84$ \\
\hline Belem, 2002 & $1.87 \pm 0.13$ & $1.61 \pm 0.14$ & $1.84 \pm 0.13$ & $1.61 \pm 0.09$ & $1.47 \pm 0.07$ & $1.49 \pm 0.06$ & $8.29 \pm 0.78$ & $9.42 \pm 1.00$ \\
\hline Montevideo, 2004 & $1.62 \pm 0.05$ & $1.66 \pm 0.06$ & $1.77 \pm 0.10$ & $1.82 \pm 0.08$ & $1.26 \pm 0.08$ & $1.34 \pm 0.07$ & $7.85 \pm 0.39$ & $8.78 \pm 0.54$ \\
\hline Mean & $1.62 \pm 0.08$ & $1.53 \pm 0.04$ & $1.68 \pm 0.07$ & $1.66 \pm 0.05$ & $1.40 \pm 0.04$ & $1.36 \pm 0.05$ & $7.94 \pm 0.25$ & $8.90 \pm 0.31$ \\
\hline Sao Tome, 2001 & $1.78 \pm 0.16$ & $1.71 \pm 0.14$ & $1.83 \pm 0.15$ & $1.74 \pm 0.11$ & $1.22 \pm 0.09$ & $1.31 \pm 0.10$ & $10.22 \pm 0.85$ & $8.79 \pm 0.77$ \\
\hline Sao Tome, 2003 & $2.08 \pm 0.13$ & $1.90 \pm 0.09$ & $2.14 \pm 0.12$ & $1.75 \pm 0.09$ & $1.30 \pm 0.07$ & $1.30 \pm 0.09$ & $10.13 \pm 0.96$ & $10.07 \pm 0.92$ \\
\hline Benin, 2000 & $1.77 \pm 0.15$ & $1.70 \pm 0.20$ & $1.83 \pm 0.15$ & $2.55 \pm 0.43$ & $1.38 \pm 0.10$ & $1.99 \pm 0.52$ & $9.11 \pm 0.71$ & $10.83 \pm 0.87$ \\
\hline Cape Town, 2002 & $1.91 \pm 0.11$ & $1.93 \pm 0.18$ & $2.04 \pm 0.10$ & $2.04 \pm 0.13$ & $1.41 \pm 0.09$ & $1.37 \pm 0.10$ & $9.57 \pm 0.55$ & $11.37 \pm 0.69$ \\
\hline Madagascar, 2003 & $1.86 \pm 0.08$ & $1.71 \pm 0.14$ & $1.98 \pm 0.15$ & $2.1 \pm 0.17$ & $1.51 \pm 0.09$ & $1.69 \pm 0.17$ & $10.21 \pm 0.86$ & $9.06 \pm 0.66$ \\
\hline Mean & $1.88 \pm 0.06$ & $1.79 \pm 0.05$ & $1.96 \pm 0.06$ & $2.04 \pm 0.15$ & $1.36 \pm 0.05$ & $1.53 \pm 0.13$ & $9.85 \pm 0.22$ & $10.02 \pm 0.50$ \\
\hline Total mean & $1.75 \pm 0.06$ & $1.66 \pm 0.05$ & $1.82 \pm 0.06$ & $1.85 \pm 0.10$ & $1.38 \pm 0.03$ & $1.45 \pm 0.07$ & $8.90 \pm 0.36$ & $9.46 \pm 0.33$ \\
\hline Intercontinental comparison ( $t$-test) & $2.62^{*}$ & $3.86^{* *}$ & $3.00^{*}$ & $2.41^{*}$ & $0.6 \operatorname{lns}$ & $1.16 \mathrm{~ns}$ & $5.66^{* * *}$ & $1.92 \mathrm{~ns}$ \\
\hline
\end{tabular}

Mean values of CVs \pm S.E. For each population, the number of values is the number of lines (see Table 1). Populations are averaged within each geographic group, and the general mean is also given. Intercontinental comparisons are made with Student's $t$-test ( $n=5$ in each group). Level of significance: ns: nonsignificant; ${ }^{*} P<0.05 ;{ }^{* *} P<0.01$; ${ }^{* *} P<0.001$.

Intraline correlations: Within each line and sex, we calculated three correlations, that is, wing-thorax, wingbristles and thorax-bristles (Table 4).

As expected from data in other species, a positive correlation was always found between wing and thorax length. In a first step, ANOVA (not shown) after a $z$ transformation revealed significant differences among populations but not between sex nor line. In a further analysis, we compared the two geographic groups by a two-way ANOVA (not shown). There was no significant effect of sex, but a significant geographic effect was found. When intercontinental comparisons are made separately for each sex, only the difference for the females is significant (Table 4). If we, however, pool the data of both sexes, the difference becomes more significant. On average, wing and thorax are less 
Table 4 Analysis of the intraline correlations

\begin{tabular}{|c|c|c|c|c|c|c|}
\hline \multirow[t]{2}{*}{ Population } & \multicolumn{2}{|c|}{ Wing-thorax } & \multicolumn{2}{|c|}{ Wing-sternopleural bristles } & \multicolumn{2}{|c|}{ Thorax-sternopleural bristles } \\
\hline & Female & Male & Female & Male & Female & Male \\
\hline Rio, 1999 & $0.53 \pm 0.06$ & $0.62 \pm 0.04$ & $0.07 \pm 0.07$ & $-0.03 \pm 0.08$ & $0.06 \pm 0.08$ & $0.005 \pm 0.078$ \\
\hline Salvador, 1999 & $0.64 \pm 0.04$ & $0.69 \pm 0.03$ & $0.01 \pm 0.12$ & $0.16 \pm 0.14$ & $0.20 \pm 0.12$ & $0.20 \pm 0.13$ \\
\hline Rio, 2000 & $0.54 \pm 0.08$ & $0.55 \pm 0.09$ & $0.10 \pm 0.11$ & $0.06 \pm 0.11$ & $0.24 \pm 0.10$ & $0.002 \pm 0.069$ \\
\hline Belem, 2002 & $0.65 \pm 0.05$ & $0.55 \pm 0.06$ & $0.04 \pm 0.09$ & $-0.07 \pm 0.12$ & $0.09 \pm 0.10$ & $0.010 \pm 0.10$ \\
\hline Montevideo, 2004 & $0.72 \pm 0.04$ & $0.70 \pm 0.04$ & $0.12 \pm 0.07$ & $0.05 \pm 0.06$ & $0.12 \pm 0.08$ & $0.10 \pm 0.07$ \\
\hline Mean & $0.62 \pm 0.04$ & $0.62 \pm 0.03$ & $0.07 \pm 0.02$ & $0.03 \pm 0.04$ & $0.14 \pm 0.03$ & $0.06 \pm 0.04$ \\
\hline Sao Tome, 2001 & $0.74 \pm 0.05$ & $0.70 \pm 0.07$ & $0.14 \pm 0.12$ & $-0.07 \pm 0.12$ & $0.24 \pm 0.12$ & $-0.04 \pm 0.12$ \\
\hline Sao Tome, 2003 & $0.80 \pm 0.05$ & $0.74 \pm 0.04$ & $-0.13 \pm-0.01$ & $-0.15 \pm 0.08$ & $-0.08 \pm 0.01$ & $-0.08 \pm 0.09$ \\
\hline Benin, 2000 & $0.70 \pm 0.05$ & $0.66 \pm 0.07$ & $-0.20 \pm 0.12$ & $-0.13 \pm 0.12$ & $0.13 \pm 0.10$ & $-0.06 \pm 0.12$ \\
\hline Cape Town, 2002 & $0.76 \pm 0.02$ & $0.75 \pm 0.05$ & $0.21 \pm 0.10$ & $0.26 \pm 0.07$ & $0.31 \pm 0.07$ & $0.29 \pm 0.09$ \\
\hline Madagascar, 2003 & $0.69 \pm 0.04$ & $0.61 \pm 0.06$ & $0.07 \pm 0.07$ & $0.01 \pm 0.08$ & $0.06 \pm 0.08$ & $0.07 \pm 0.08$ \\
\hline Mean & $0.74 \pm 0.02$ & $0.69 \pm 0.03$ & $0.02 \pm 0.08$ & $-0.02 \pm 0.07$ & $0.13 \pm 0.03$ & $0.04 \pm 0.07$ \\
\hline Total mean & $0.68 \pm 0.03$ & $0.66 \pm 0.02$ & $0.04 \pm 0.04$ & $0.01 \pm 0.04$ & $0.14 \pm 0.04$ & $0.05 \pm 0.04$ \\
\hline $\begin{array}{l}\text { Intercontinental comparison } \\
(t \text {-test) }\end{array}$ & $2.97^{*}$ & $1.68 \mathrm{~ns}$ & $0.62 \mathrm{~ns}$ & $0.59 \mathrm{~ns}$ & $0.13 \mathrm{~ns}$ & $0.35 \mathrm{~ns}$ \\
\hline
\end{tabular}

For each population, an average value of $r$ coefficient is calculated based on the respective number of lines. For each geographic group, the S.E. is calculated from the mean values of the five populations. Level of significance: ns: non significant; ${ }^{*} P<0.05$.

correlated in American populations $(r=0.62 \pm 0.02$; average of female and male values: $n=10)$ than in African ones $(r=0.72 \pm 0.02 ; n=10, P<0.01)$. Correlation between wing and STP bristles was always low, and the overall value $(r=0.02 \pm 0.03 ; n=20)$ is not different from zero. A slightly higher and significant correlation $(r=0.10 \pm 0.03 ; n=20)$ was observed between thorax length and STP bristles number: a larger thorax is accompanied by a slight increase in thoracic bristles number.

Relationship between intraline CVs and wing-thorax correlation: Previous results have shown that populations were significantly heterogeneous, both for the intraline CVs and for the intraline correlation between wing and thorax. More precisely, American populations are consistently less variable and their wing-thorax correlation is smaller than in African populations.

Variability among individuals of the same line reflects mostly an environmental component (David et al, 2005). For instance, if for an unknown reason (eg development in a drier part of a vial) an individual becomes smaller, this phenomenon should increase both the variance in size and the covariance between size traits. We asked the question: is there a positive relationship, at the line level, between the CVs for size traits and the wing-thorax correlation? For estimating an overall CV for size, we averaged for each line the values found for wing and thorax. For each population, we calculated the correlation between the intraline $\mathrm{CV}$ and the intraline wingthorax, $r$ coefficient. The answer to the question was clearly yes. Average values for the 10 populations are $r=0.55 \pm 0.07$ in females and $r=0.39 \pm 0.09$ in males. This phenomenon is illustrated graphically (Figure 2) for the 34 lines that compose the two Rio samples. If we first consider the relationship between body size $\mathrm{CV}$ and wing-thorax Pearson's coefficients (Figure 2a), there is a positive correlation between the two variables, but the relationship is better described by a quadratic polynomial, as shown in the figure, and both the linear $(P=0.002)$ and the quadratic $(P=0.04)$ components are significant. This curvilinear regression disappears however by using the $z$ transformation (Figure $2 \mathrm{~b}$ ). In this case, the quadratic parameter is no more significant $(P=0.35)$ while the linear component becomes highly significant $(P<0.001)$.

We also analysed the same relationship among population mean values, pooling into a single sample the American and African populations. We again found a significant, positive correlation between the two kinds of parameters (Figure 3). As suggested above, a higher phenotypic variability of size traits is always accompanied by a stronger positive correlation between these traits. In this case, the overall positive regression is mainly explained by the difference between American and African populations.

\section{Genetic variability}

ICC and genetic CV: ICC coefficients are given in Table 5. A two-way (population and sex) ANOVA (not shown) applied on the total set for each trait evidenced a significant population effect for wing length, thorax length and STP bristle number, and also a significant sex difference for STP. Further analyses did not however show any significant difference between the two geographic groups, although in most cases, average ICCs were slightly higher in the American group (see Table 5). Average values among traits are quite similar and never exceed 0.30. Similar values were already observed in an Indian population (Karan et al, 1999a).

We also calculated for each trait the genetic CVs (not shown) and did not find any significant difference between sexes. Also, differences between the two geographic groups were never significant. There were however significant differences between traits. Average values $(n=20)$ were almost identical for size traits, that is $1.10 \pm 0.07$ and $1.07 \pm 0.06$ for wing and thorax 

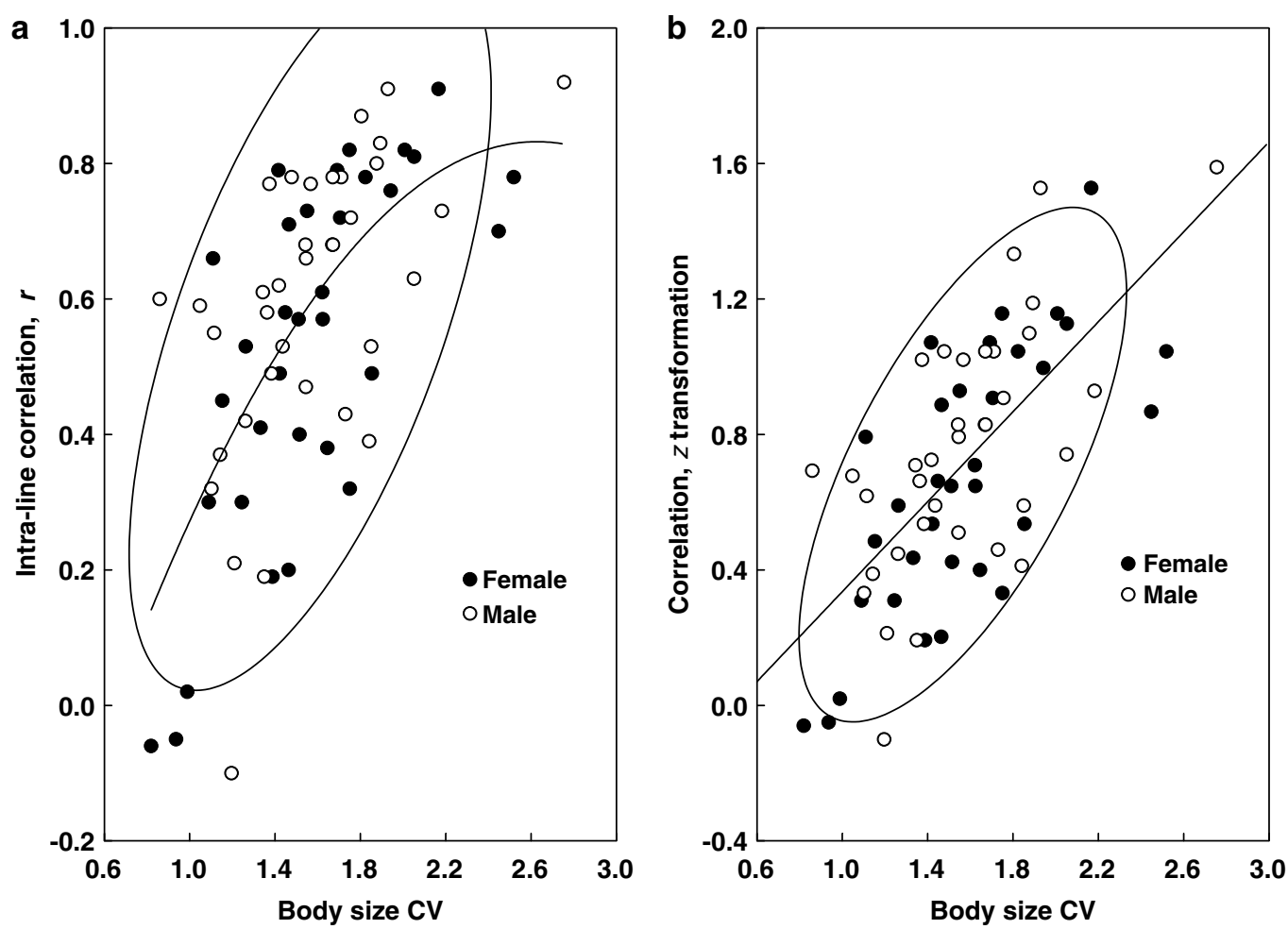

Figure 2 Relationship between the intraline CV of body size (average for wing length and thorax length) and wing-thorax correlation. Results of 34 lines from Rio (female and male data pooled). Each point corresponds to an isofemale line. (a) Pearson's coefficient $r$; ellipse of $90 \%$ is shown, and also a significant curvilinear adjustment. (b) $z$ transformation of $r$. The curvilinear component becomes non significant, and a linear regression is shown.

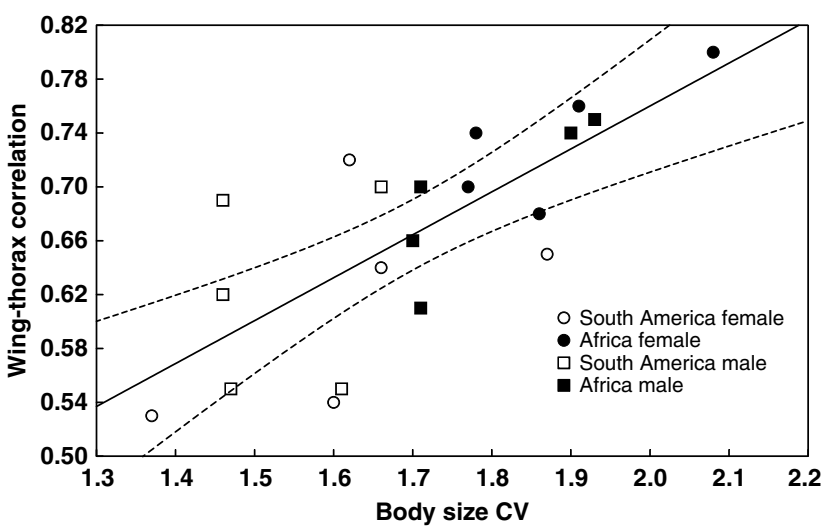

Figure 3 Relationship, at the population level, between the intraline variability of body size (average $\mathrm{CV}$ for wing and thorax length) and wing-thorax intraline correlation. Each value corresponds to a population. Overall correlation is $r=0.75$; the slope of the regression $b=0.322 \pm 0.067, P<0.001$ (pooled data for females and males).

length respectively; they were significantly less for $\mathrm{W} / \mathrm{T}$ ratio $(0.89 \pm 0.06)$ and much bigger for STP number $(4.69 \pm 0.36)$.

Genetic correlations: Genetic correlations, among family means, were calculated within each population (data not shown). Between wing and thorax length, positive significant correlations were found in all cases, with no difference between groups or sexes, and the overall value is $0.66 \pm 0.04(n=20)$. Correlations between
STP bristles and size traits were close to zero and not significant: average values were, for wing-bristles, $r=-0.01 \pm 0.06$, and for thorax-bristles, $r=-0.06 \pm 0.06$ ( $n=20$ in each case). We also asked the question: is there a relationship, at the population level, between genetic variability $(\mathrm{CVg})$ and genetic correlation between wing and thorax. Data (not shown) produced quite unexpected results. In males, the correlation was $r=0.61$ $(n=10)$, not very far from the significance threshold $(0.63$ for $P=0.05)$. In females, however, the correlation was negative $(r=-0.17)$ and close to zero. Such a contrast deserves further investigation. A conservative hypothesis is that the correlation, if any, is low when populations are compared. while it is highly significant when the intraline variability is considered (Figures 2 and 3).

Phenotypic variability according to latitude of origin Besides the 10 populations that were investigated with the ILT technique (see Table 1), we also had data for six African and one Indian population (Chandigarh) that were measured on mass laboratory strain (Table 6). We can also compare these results to those of 10 mass populations from India, already analysed in a previous paper (Karan et al, 2000).

For each geographic group (American, African and Indian), we investigated the relationship between each quantitative trait and latitude of origin (see Table 7). In most cases, a positive correlation was found with latitude of origin, but a significant value was observed, 
Table 5 Intraclass correlation coefficient (ICC) in the 10 populations investigated with the isofemale line technique

\begin{tabular}{|c|c|c|c|c|c|c|c|c|}
\hline \multirow[t]{2}{*}{ Population } & \multicolumn{2}{|c|}{ Wing length } & \multicolumn{2}{|c|}{ Thorax length } & \multicolumn{2}{|c|}{ Wing/thorax ratio } & \multicolumn{2}{|c|}{ Sternopleural bristles } \\
\hline & Female & Male & Female & Male & Female & Male & Female & Male \\
\hline Rio, 1999 & 0.15 & 0.18 & 0.11 & 0.13 & 0.12 & 0.14 & 0.32 & 0.24 \\
\hline Salvador, 1999 & 0.42 & 0.31 & 0.45 & 0.21 & 0.59 & 0.40 & 0.16 & 0.03 \\
\hline Rio, 2000 & 0.22 & 0.30 & 0.34 & 0.43 & 0.39 & 0.27 & 0.34 & 0.15 \\
\hline Belem, 2002 & 0.36 & 0.37 & 0.26 & 0.29 & 0.23 & 0.19 & 0.32 & 0.17 \\
\hline Montevideo, 2004 & 0.34 & 0.37 & 0.23 & 0.30 & 0.24 & 0.20 & 0.16 & 0.15 \\
\hline Mean (American) & $0.30 \pm 0.05$ & $0.31 \pm 0.03$ & $0.28 \pm 0.06$ & $0.27 \pm 0.05$ & $0.31 \pm 0.08$ & $0.24 \pm 0.05$ & $0.26 \pm 0.04$ & $0.15 \pm 0.03$ \\
\hline Sao Tome, 2001 & 0.29 & 0.51 & 0.20 & 0.37 & 0.21 & 0.30 & 0.40 & 0.32 \\
\hline Sao Tome, 2003 & 0.20 & 0.19 & 0.25 & 0.20 & 0.31 & 0.37 & 0.21 & 0.22 \\
\hline Benin, 2000 & 0.11 & 0.09 & 0.37 & 0.11 & 0.38 & 0.11 & 0.07 & 0.07 \\
\hline Cape Town, 2002 & 0.18 & 0.24 & 0.12 & 0.14 & 0.19 & 0.14 & 0.28 & 0.15 \\
\hline Madagascar, 2003 & 0.35 & 0.38 & 0.29 & 0.18 & 0.35 & 0.30 & 0.15 & 0.10 \\
\hline Mean (African) & $0.23 \pm 0.04$ & $0.28 \pm 0.07$ & $0.25 \pm 0.04$ & $0.20 \pm 0.05$ & $0.29 \pm 0.04$ & $0.24 \pm 0.05$ & $0.22 \pm 0.06$ & $0.17 \pm 0.04$ \\
\hline Total mean & $0.26 \pm 0.03$ & $0.29 \pm 0.04$ & $0.26 \pm 0.03$ & $0.24 \pm 0.03$ & $0.30 \pm 0.04$ & $0.24 \pm 0.03$ & $0.24 \pm 0.03$ & $0.16 \pm 0.03$ \\
\hline
\end{tabular}

Table 6 Values of morphometrical traits measured on mass populations from Africa or India

\begin{tabular}{|c|c|c|c|c|c|c|c|}
\hline \multirow[t]{2}{*}{ Origin } & \multirow[t]{2}{*}{ Latitude } & \multicolumn{2}{|c|}{ Wing } & \multicolumn{2}{|c|}{ Thorax } & \multicolumn{2}{|c|}{ Sternopleural } \\
\hline & & Female & Male & Female & Male & Female & Male \\
\hline Egypt (Alexandria) & 31.1 & $290.9 \pm 1.09$ & $286.7 \pm 1.03$ & $133.3 \pm 0.55$ & $133.0 \pm 0.51$ & $21.4 \pm 0.36$ & $20.0 \pm 0.33$ \\
\hline Portugal (Madeira) & 32.4 & $295.8 \pm 1.09$ & $288.0 \pm 0.76$ & $130.7 \pm 0.55$ & $130.7 \pm 0.48$ & $18.4 \pm 0.32$ & $17.4 \pm 0.23$ \\
\hline Ivory Coast (Abidjan) & 5.3 & $282.1 \pm 0.87$ & $278.6 \pm 1.26$ & $130.5 \pm 0.52$ & $130.3 \pm 0.56$ & $21.8 \pm 0.41$ & $20.0 \pm 0.41$ \\
\hline Congo (Pointe Noire) & 4.5 & $279.8 \pm 1.32$ & $282.9 \pm 1.34$ & $131.2 \pm 0.54$ & $132.5 \pm 0.62$ & $21.2 \pm 0.36$ & $18.5 \pm 0.36$ \\
\hline Congo (Brazzaville) & 1.0 & $285.1 \pm 1.43$ & $282.7 \pm 1.53$ & $129.3 \pm 0.79$ & $129.8 \pm 0.79$ & $18.1 \pm 0.40$ & $16.8 \pm 0.43$ \\
\hline Benin (Cotonou) & 6.2 & $280.5 \pm 1.60$ & $274.9 \pm 1.68$ & $129.6 \pm 0.71$ & $127.9 \pm 0.63$ & $18.9 \pm 0.55$ & $18.9 \pm 0.56$ \\
\hline India (Chandigarh) & 30.5 & $289.0 \pm 0.87$ & $281.0 \pm 1.07$ & $136.2 \pm 0.58$ & $133.4 \pm 0.51$ & $21.0 \pm 0.32$ & $20.0 \pm 0.29$ \\
\hline
\end{tabular}

The number of observations is always equal or superior to 25 flies.

Table 7 Relationship between quantitative traits and latitude of origin in three geographic groups

\begin{tabular}{|c|c|c|c|c|c|c|c|}
\hline \multirow[t]{2}{*}{ Geographic group } & \multirow[t]{2}{*}{ Trait } & \multicolumn{3}{|c|}{ Female } & \multicolumn{3}{|c|}{ Male } \\
\hline & & $a$ & $b$ & $r$ & $a$ & $b$ & $r$ \\
\hline \multirow{4}{*}{ America $(n=5)$} & Wing & $297.56 \pm 2.66$ & $0.037 \pm 0.122$ & 0.17 & $291.76 \pm 2.42$ & $0.047 \pm 0.111$ & 0.24 \\
\hline & Thorax & $137.43 \pm 0.45$ & $-0.005 \pm 0.021$ & -0.14 & $136.52 \pm 0.66$ & $-0.011 \pm 0.030$ & -0.21 \\
\hline & Wing/thorax ratio & $2.165 \pm 0.015$ & $0.0003 \pm 0.0007$ & 0.27 & $2.137 \pm 0.013$ & $0.0005 \pm 0.0005$ & 0.46 \\
\hline & Sternopleural bristles & $19.34 \pm 0.15$ & $0.0107 \pm 0.0070$ & 0.66 & $18.58 \pm 0.10$ & $-0.0055 \pm 0.0047$ & -0.56 \\
\hline \multirow{4}{*}{ Africa $(n=11)$} & Wing & $278.95 \pm 1.82$ & $0.335 \pm 0.100^{* *}$ & 0.74 & $275.79 \pm 1.97$ & $0.271 \pm 0.109^{*}$ & 0.64 \\
\hline & Thorax & $130.57 \pm 0.55$ & $0.0026 \pm 0.031$ & 0.03 & $129.92 \pm 0.71$ & $-0.0019 \pm 0.039$ & -0.02 \\
\hline & Wing/thorax ratio & $2.137 \pm 0.016$ & $0.0025 \pm 0.0008^{*}$ & 0.70 & $2.122 \pm 0.013$ & $0.0021 \pm 0.0007^{*}$ & 0.71 \\
\hline & STP & $20.08 \pm 0.50$ & $-0.0147 \pm 0.0277$ & -0.17 & $18.55 \pm 0.42$ & $0.0038 \pm 0.023$ & 0.05 \\
\hline \multirow[t]{4}{*}{ India $(n=11)$} & Wing & $243.37 \pm 5.73$ & $1.281 \pm 0.228^{* * *}$ & 0.88 & $239.16 \pm 9.66$ & $1.196 \pm 0.386^{*}$ & 0.72 \\
\hline & Thorax & $115.11 \pm 3.37$ & $0.511 \pm 0.147^{* *}$ & 0.76 & $114.11 \pm 4.59$ & $0.477 \pm 0.183^{*}$ & 0.66 \\
\hline & Wing/thorax ratio & $2.116 \pm 0.030$ & $0.0014 \pm 0.0012$ & 0.39 & $2.098 \pm 0.032$ & $0.0015 \pm 0.0013$ & 0.36 \\
\hline & STP & $22.17 \pm 1.85$ & $0.0088 \pm 0.0739$ & 0.04 & $21.89 \pm 2.02$ & $-0.0544 \pm 0.0806$ & 0.22 \\
\hline
\end{tabular}

$a$ : intercept; $b$ : linear regression coefficient; $r$ : correlation coefficient. Values of $b$ significantly different from zero are marked. Level of significance: ${ }^{*} P<0.05 ;{ }^{* *} P<0.01$; ${ }^{* * *} P<0.001$.

for both sexes, in four cases only: wing and thorax length in India, and wing length and $\mathrm{W} / \mathrm{T}$ ratio in Africa. Interestingly, no significant effect was detected in American populations, for which the regression coefficients were always close to zero. These results are illustrated in Figure 4, for wing length and $\mathrm{W} / \mathrm{T}$ ratio in females.
The graph for wing length clearly evidences a steep cline on the Indian continent, a smooth cline in Africa and the complete lack of clinal variation on the American continent. On average, American populations are bigger, Indian are much smaller and African are intermediate. Differences are more pronounced at the intercept level (the Equator), and values converge at higher latitudes. 

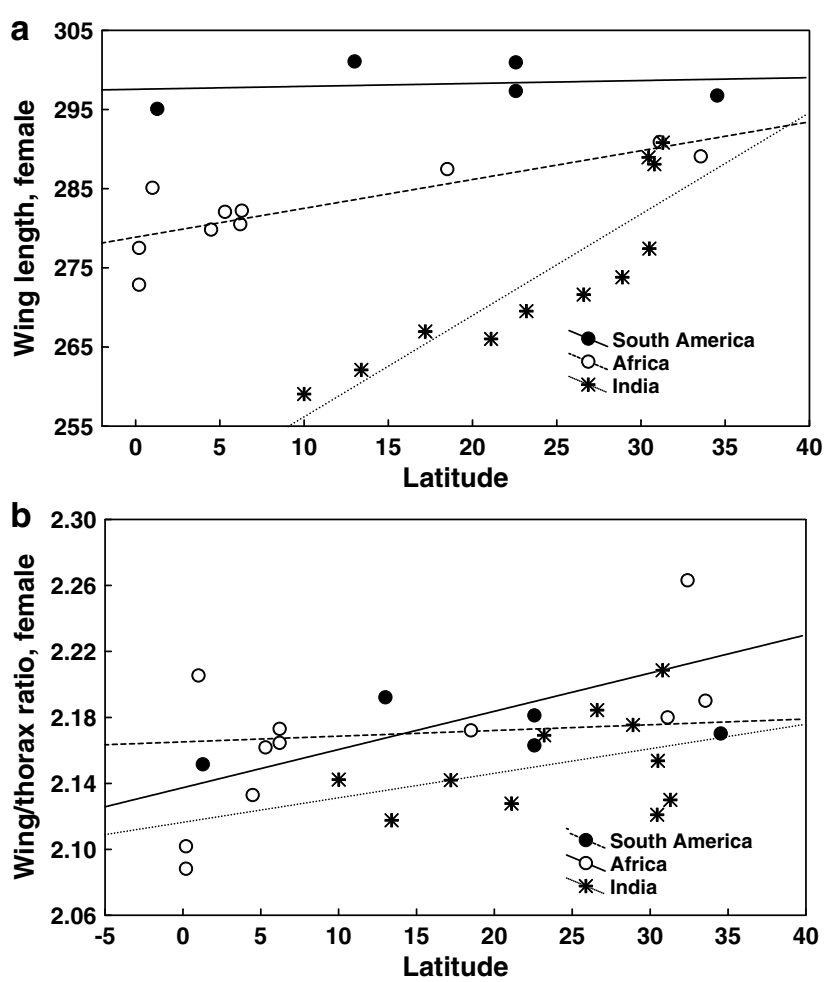

Figure 4 Relationship between latitude of origin and wing length or wing/thorax ratio in Zaprionus females. (a) Latitudinal clinal variations are significant for African and Indian populations, but not for American ones. Differences among geographic groups are more pronounced at the Equator level. (b) A latitudinal trend seems to exist for wing/thorax ratio in India and South America but is significant only for Indian populations. When all populations are pooled, an overall significant increase with latitude is detected $(b=0.0012 \pm 0.0006, P<0.04)$

A somehow different picture is obtained for the $\mathrm{W} / \mathrm{T}$ ratio. A general observation is the broad dispersal of the mean values. The only significant increase with latitude (Table 7) is found for the African populations. In that case, however, there are two populations that are quite apart from the regression line. At the equator level, the population of Brazzaville exhibits a high value (2.20); at a latitude of $32.4^{\circ}$, the population of Madeira has a still higher ratio (2.26). Interestingly, these two values were obtained on mass cultures kept several years in the laboratory. It is thus possible that $\mathrm{W} / \mathrm{T}$ ratio is more sensitive to laboratory genetic drift than the traits themselves. If we consider only the five African populations analysed with the ILT method (Table 1), the correlation of $\mathrm{W} / \mathrm{T}$ ratio with latitude is 0.81 in female and 0.79 in male, while the significance threshold is 0.87 for $P=0.05$. Finally, if we pool all populations into a single sample $(n=27)$, the relationship with latitude becomes significant: female $r=0.41(P=0.034)$; male $r=0.39(P=0.042)$ (see Table 7).

We may conclude that a latitudinal trend for an increased $\mathrm{W} / \mathrm{T}$ ratio (and a lesser wing loading) exists in Z. indianus, although many more populations should be investigated immediately after being collected for getting a better geographic picture.

\section{Discussion}

The major aim of this paper was the analysis of two kinds of quantitative traits (body size and thoracic bristle number) in the recently introduced South-American populations of Z. indianus, and to compare the results to those of ancestral populations in the Old World. All American samples were remarkable by their big size. Although there were slight differences among geographic samples, there was no trend for a latitudinal clinal variation. Latitudinal clines, however, exist in India for wing and thorax length, and in Africa for wing length and wing/thorax ratio. Such trends agree with what is known in other cosmopolitan species, such as $D$. melanogaster and D. simulans (Capy et al, 1993; Gibert et al, 2004), D. kikkawai (Karan et al, 1998b) or D. subobscura (Huey et al, 2000).

Tropical Africa harbours the ancestral populations of Z. indianus (Tsacas, 1985), while Indian populations have been introduced, at an unknown date, but certainly more than three decades ago (Gupta, 1970). In this respect, it is at first sight surprising that latitudinal clines are more pronounced in India than in Africa. Such an observation is however not unique, and a similar case exists in $D$. melanogaster for chromosome rearrangements polymorphism. In tropical Africa, ancestral populations are highly variable for inversion frequencies, but with no latitudinal trend (Aulard et al, 2002). In introduced populations, however, cosmopolitan inversions exhibit clear latitudinal clines, for example in North America or Australia (Knibb, 1982; Lemeunier and Aulard, 1992). It is possible that a founder event, followed by a rapid extension, renders the populations more sensitive to climatic selective pressures. In the America-introduced D. subobscura populations, latitudinal clines were observed, quite rapidly, for chromosome inversions (Prevosti et al, 1985; Ayala et al, 1989), while it took more than 10 years, for the same populations, to show a latitudinal cline for wing length (Huey et al, 2000). The absence of latitudinal differentiation of body size in American Z. indianus is presumably a consequence of a too short evolutionary time.

Contrary to body size, the sternopleural bristle number revealed only a small amount of between-population differentiation and, more importantly, no significant influence of latitude, in any part of the world. Sternopleural bristle number shows clear latitudinal clines in $D$. melanogaster and, to a lesser degree, in $D$. simulans (Capy et al, 1993; Gibert et al, 2004), while there is no cline in D. kikkawai (Karan et al, 1998b). The adaptive significance of STP number is difficult to understand (Moreteau et al, 2003), and it is possible that significant differences exist among species, concerning the reactivity of this trait to climatic conditions.

The geographic origin of the propagule that colonized Brazil in 1998 is not known, but our data help to discuss the matter. The big size of American flies suggests a highlatitude origin. In this respect the biggest population investigated so far from the Afrotropical region is from Madeira island. This population, however, corresponds to a fairly recent introduction. Z. indianus was absent of Madeira at the time D. madeirensis was described from this island (Monclús, 1984). A more detailed analysis of populations on African mainland, for example from Sénégal, remains to be undertaken. 
Our results have shown that the genetic variability of the American populations, estimated either with the intraclass correlation or the genetic $\mathrm{CV}$, was quite similar to what has been found in African populations for which a large genetic size is expected. This suggests that the American colonizing propagule was of a sufficient size to carry practically all the genetic diversity of the origin population. A similar conclusion was already obtained from a comparison of allozvme diversity in Brazilian and Old-World populations (Mattos Machado et al, in press).

We, however, made an unexpected observation: the occurrence of a significant difference in the intraline variability between American and African populations. The intraline CV mostly expresses an environmental component of the phenotypic variance (David, 2005). The fact that American populations were consistently less variable, for size traits in both sexes and for STP bristle number in females, is difficult to explain. The phenomenon cannot be due to uncontrolled variations in the experimental conditions (eg food quality), since measurements were made in four different years, and at the same time for American and African samples. The data might be due to a lesser genetic variability in the American flies, related to the foundation. However, the CVgs for size traits are almost identical: $1.07 \pm 0.01$ in America and $1.10 \pm 0.04$ in Africa ( $n=4$ in each case). We may therefore propose that the genetic difference between American and African populations concerns the level of developmental stability or canalization. Why and how American flies are phenotypically more stable remains, however, a matter of speculation.

The lesser phenotypic variability of the American flies is accompanied by a lesser correlation between wing and thorax. This result is clearly visible at the line level within a given population: the most variable lines are also those where the wing-thorax correlation is maximum. An analogous observation (unpublished results) has also been made in D. melanogaster, so that the phenomenon might be a general characteristics in Drosophila. We also found a similar relationship at the populations level (Figure 2) contrasting the American populations with a lower variability and the African ones. We also asked the question: are the intra-line CVs and the between-line (genetic) CVs correlated? These correlations were calculated separately for each sex and trait $(n=8)$ on the 10 populations from America and Africa. Values of $r$ ranged between -0.03 and 0.44 . with an overall mean of $0.21 \pm 0.06$. This mean is significantly greater than zero, although the coefficient of determination $\left(R^{2}=0.04\right)$ is very small. The fact that the withinand the between-line variability are almost independent is a further argument to say that they measure different components of the total phenotypic variance (David et al, 2005).

Our colonization scenario of South America is at least plausible: a single, fairly large propagule was introduced from an Afrotropical country, followed by a rapid geographic spread, but several questions remain. One is the process of geographic extension in America, with two likely possibilities: a spontaneous and progressive diffusion from the margins of natural populations; or jumps from a locality to a distant one, thanks to an artificial, man-made transportation. The role of such transportations within Brazil may be important. It is presumably the cause of the presence of $Z$. indianus in oceanic islands which, like Fernando de Noronha island, are at a distance of $350 \mathrm{~km}$ from the mainland (BC BitnerMathé, unpublished). Another point concerns the geographic limits of Zaprionus populations. Going south towards high latitudes, Z. indianus is found in Uruguay, but only during the warm season (Goñi et al, 2001, unpublished). Little doubt remains that winter, cold temperature is responsible of the absence of permanent populations. Whether these marginal populations will eventually be able to increase their cold tolerance and make permanent populations should be a subject of future investigations. Going north of the Equator, favourable tropical climatic conditions are found up to southern USA. Indeed, Florida harbours at least two circumtropical species, D. ananassae and D. malerkotliana (Birdsley, 2003; Medeiros et al, 2003), that are absent from Uruguay, but present in Southern Brazil. In other words, climatic conditions in Florida are more favourable to tropical species than in Uruguay. Owing to the capacity of $Z$. indianus to travel with fruit shipments, we may expect that North America will be colonized in a short future, the climatic limit being at the level of southern USA states. Ecological surveys are strongly needed in all North-American countries.

\section{Acknowledgements}

We thank Drs M Boulétreau, M-L Cariou and D Lachaise for providing the wild living samples from South Africa, Bénin and Sao Tomé, respectively. This work was supported by the Comité Français d'Evaluation de la Coopération Universitaire avec le Brésil (COFECUB) and the Coordenação de Aperfeicoamento de Pessaoal de Nivel Superior (CAPES). It is paper no 3 of a FrenchBrazilian cooperation programme on Zaprionus.

\section{References}

Andrewartha HG, Birch LC (1954). The Distribution and Abundance of Animals. University of Chicago Press: Chicago.

Angilletta MJ, Dunham AE (2003). The temperature-size rule in ectotherms: simple evolutionary explanations may not be general. Am Nat 162: 332-342.

Atkinson D, Sibly RM (1997). Why are organisms usually bigger in colder environments? Making sense of a life history puzzle. Trends Ecol Evol 12: 235-239.

Aulard S, David JR, Lemeunier F (2002). Chromosomal inversion polymorphism in tropical populations of Drosophila melanogaster. Genet Res 79: 49-63.

Ayala FJ, Serra L, Prevosti A (1989). A grand experiment in evolution: the Drosophila subobscura colonization of the Americas. Genome 31: 246-255.

Bijlsma R, Loeschcke V (1997). Environmental Stress, Adaptation and Evolution. Birkhäuser Verlag: Basel.

Birdsley JS (2003). Drosophila malerkotliana and D. ananassae in Florida. Drosophila Inf Serv 86: 112.

Blackburn TM, Gaston KJ, Loder N (1999). Geographic gradients in body size: a clarification of Bergmann's rule. Divers Distributions 5: 165-174.

Brncic D, Prevosti A, Budnik M, Monclús M, Ocaña J (1981). Colonization of Drosophila subobscura in Chile. I. First population and cytogenetics studies. Genetica 56: 3-9.

Capy P, Pla E, David JR (1993). Phenotypic and genetic variability of morphometrical traits in natural populations of Drosophila melanogaster and D. simulans. I. Geographic variations. Genet Sel Evol 25: 517-536. 
Chassagnard MT, Kraaijeveld AR (1991). The occurrence of Zaprionus sensu stricto in the Palearctic region (Diptera: Drosophilidae). Annls Soc Ent Fr 27: 495-496.

Conner JK, Hartl DL (2004). A Primer of Ecological Genetics. Sinauer Associates Inc.: Sunderland, MA, USA.

Cossins AR, Bowler K (1987). Temperature Biology of Animals. Chapman \& Hall: London.

David J, Clavel MF (1965). Interaction entre le génotype et le milieu d'élevage. Conséquences sur les caractéristique du développement de la Drosophile. Bull Biol Fr Belg 99: 369-378.

David JR, Allemand R, Capy P, Chakir M, Gibert P, Pétavy G et al (2004). Comparative life histories and ecophysiology of Drosophila melanogaster and D. Simulans. Genetica 120: 151-163.

David JR, Capy P (1988). Genetic variation of Drosophila melanogaster natural populations. TIG 4: 106-111.

David JR, Gibert P, Legout H, Capy P, Moreteau B (2005). Isofemale lines in Drosophila: an empirical approach to quantitative traits analysis in natural populations. Heredity 94: 3-12.

David JR, Tsacas L (1981). Cosmopolitan subcosmopolitan and widespread species: different strategies within the Drosophilid family (Diptera). CR Soc Biol 57: 11-26.

Gibert P, Capy P, Imasheva A, Moreteau B, Morin JP, Pétavy G et al (2004). Comparative analysis of morphometrical traits among Drosophila melanogaster and D. simulans: genetic variability, clines and phenotypic plasticity. Genetica 120: 165-179.

Goñi B, Fresia P, Calviño M, Ferreiro MJ, Valente VLS, Basso da Silva L (2001). First record of Zaprionus indianus Gupta, 1970 (Diptera Drosophilidae) in southern localities of Uruguay, South America. Drosophila Inf Serv 84: 61-65.

Gupta JP (1970). Description of a new species of Phorticella zaprionus (Drosophilidae) from India. Proc Ind Natl Sci Acad 36: 62

Hoffmann AA, Parsons PA (1988). The analysis of quantitative variation in natural populations with isofemale strains. Genet Sel Evol 20: 87-98.

Hoffmann AA, Parsons PA (1991). Evolutionary Genetics and Environmental Stress. Oxford University Press: Oxford.

Hoffmann AA, Parsons PA (1997). Extreme Environmental Change and Evolution. Cambridge University Press: Cambridge.

Houle D (1992). Comparing evolvability and variability of quantitative traits. Genetics 130: 195-204.

Huey RB, Gilchrist GW, Carlson ML, Berrigan D, Serra L (2000). Rapid evolution of a geographic cline in size in an introduced fly. Science 287: 308-309.

Karan D, Dahiya N, Munjal AK, Gibert P, Moreteau B, Parkash R, David JR (1998a). Desiccation and starvation tolerance of adult Drosophila: opposite latitudinal clines in natural populations of three different species. Evolution 52: 825-831.

Karan D, Munjal AK, Gibert P, Moreteau B, Parkash R, David JR (1998b). Latitudinal clines for morphometrical traits in Drosophila kikkawai: a study of natural populations from the Indian subcontinent. Genet Res Camb 71: 31-38.

Karan D, Moreteau B, David JR (1999a). Growth temperature and reaction norms of morphometrical traits in a tropical drosophilid: Zaprionus indianus. Heredity 83: 398-407.

Karan D, Morin JP, Gravot E, Moreteau B, David JR (1999b). Body size reaction norms in Drosophila melanogaster: temporal stability and genetic architecture in a natural population. Genet Sel Evol 31: 491-508.
Karan D, Dubey S, Moreteau B, Parkash R, David JR (2000). Geographical clines for quantitative traits in natural populations of a tropical drosophilid: Zaprionus indianus. Genetica 108: $91-100$.

Knibb WR (1982). Chromosome inversion polymorphisms in Drosophila melanogaster. II. Geographic clines and climatic associations in Australasia, North America and Asia. Genetica 58: $213-221$.

Leather S, Walters K, Bale J (1993). The Ecology of Insects Overwintering. University Press Cambridge: Cambridge.

Lemeunier F, Aulard S (1992). Inversion polymorphism in Drosophila melanogaster. In: Krimbas CB, Powell JR (eds) Drosophila Inversion Polymorphism. CRC Press: Boca Raton. pp 339-405.

Mattos Machado T, Solé-Cava A, David JR, Bitner-Mathé BC (2005). Allozyme variability in an invasive drosophilid, Zaprionus indianus (Diptera: Drosophilidae): comparison of a recently introduced Brazilian population with Old World populations. Annls Soc Ent Fr (NS) (in press).

Medeiros HF, Martins MB, David JR (2003). The extension of the geographic range of Drosophila malerkotliana on the American continent. Drosophila Inf Serv 86: 89.

Monclús M (1984). Drosophilidae of Madeira, with the description of Drosophila madeirensis, n.sp. Z Zool Syst Evolutions Forsch 22: 94-103.

Moreteau B, Gibert P, Delpuech JM, Pétavy G, David JR (2003). Phenotypic plasticity of sternopleural bristle number in temperate and tropical populations of Drosophila melanogaster. Genet Res Camb 81: 25-32.

Partridge L, Coyne JA (1997). Bergmann's rule in ectotherms: is it adaptive? Evolution 51: 632-635.

Partridge L, French V (1996). Why get bigger in the cold? In: Johnston IA, Bennett AB (eds) Animals and Temperature. Cambridge University Press: Cambridge. pp 265-292.

Pétavy G, Morin JP, Moreteau B, David JR (1997). Growth temperature and phenotypic plasticity in two Drosophila sibling species: probable adaptive changes in flight capacities. J Evol Biol 10: 875-887.

Precht H, Christophersen J, Hensel H, Larcher W (1973). Temperature and Life. Springer Verlag: Berlin.

Prevosti A, Serra L, Ribó G, Aguadé M, Sagarra E, Monclús M et al (1985). Colonization of Drosophila subobscura in Chile. II. Clines in the chromosomal arrangements. Evolution 39: 838-844.

Santos M, Céspedes W, Balanyà J, Trotta V, Calboli FCF (2005). Temperature-related genetic changes in laboratory populations of Drosophila subobscura: evidence against simple climatic-based explanations for latitudinal clines. Am Nat 165: 258-273.

Statsoft (1999). Statistica (data analysis software system). Version 5.5.

Tidon R, Leite DF, Leão BFD (2003). Impact of the colonization of Zaprionus (Diptera, Drosophilidae) in different ecosystems of the Neotropical Region: 2 years after the invasion. Biol Cons 112: 299-305.

Tsacas L (1985). Zaprionus indianus Gupta, 1970 nouveau nom pour le plus commun des Zaprionus africains (Diptera, Drosophilidae). Annls Soc ent Fr (NS) 21: 343-344.

Van Voorhies WA (1996). Bergmann size clines: a simple explanation for their occurrence in ectotherms. Evolution 50: 1259-1264.

Vilela CR (1999). Is Zaprionus indianus Gupta, 1970 (Diptera, Drosophilidae) currently colonizing the Neotropical region? Drosophila Inf Serv 82: 37-39. 